\title{
Resilience, risk, mental health and well-being: associations and conceptual differences
}

\author{
Frauke Schultze-Lutter $^{1} \cdot$ Benno G. Schimmelmann ${ }^{1} \cdot$ Stefanie J. Schmidt $^{1}$
}

Published online: 22 April 2016

(C) Springer-Verlag Berlin Heidelberg 2016

Resilience and well-being have become commonplace terms in a wide range of scientific as well as political mental health contexts and are now rapidly spreading within the child and adolescent psychiatric literature. For example, PubMed lists 751 articles published in European Child \& Adolescent Psychiatry including either term, and after a slow steady increase between 1993 and 2014, the number of occurrences recently jumped from 63 in 2014 to 116 in 2015 (Fig. 1). However, while frequently debated, the number of papers directly addressing these two concepts (indicated by their appearance in title and/or abstract) is far lower, with only six papers [1-6] carrying either term in their title (Fig. 1). This lack of research addressing resilience and well-being is accompanied by-or likely resulting in-a lack of common understanding of these terms.

A search of the mental health publications of the World Health Organisation (WHO) exemplifies this lack of common understanding of these terms. In the early 2000s, resilience was broadly defined as 'resistance to disease' ${ }^{1}$ [7, $\mathrm{p}$. 27] or 'the capacity to cope with adversity and to avoid

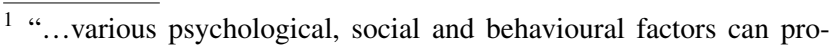
tect health and support positive mental health. Such protection facilitates resistance (resilience) to disease, minimizes and delays the emergence of disabilities and promotes more rapid recovery from illness." [7, p. 27].
}

Frauke Schultze-Lutter

frauke.schultze-lutter@kjp.unibe.ch

1 University Hospital of Child and Adolescent Psychiatry and Psychotherapy, University of Bern, Bolligerstrasse 111, 3000 Bern 60, Switzerland breakdown when confronted by stressors' ${ }^{2}$ [8, p. 20]. Here, resilience equals or is a part of personal protective factors ${ }^{3}$ and functions as a condition or promoter of mental health. In contrast, mental health has been more recently regarded not as a possible consequence but as 'a fundamental element ${ }^{4}$ of resilience [9, p. III], or, alternatively, 'as a state of well-being in which every individual ... can cope with the normal stresses of life ${ }^{5}$ [10], the latter making resilience a feature of mental health.

In light of these examples, it is hardly surprising that reviews on the concepts of resilience or well-being have

\footnotetext{
2 "Resilience. The capacity to cope with adversity and to avoid breakdown when confronted by stressors differs tremendously among individuals. Not all responses to stress are pathological and they may serve as coping mechanisms. Numerous researchers have studied healthy mechanisms of defence and coping. Rutter [12] conceived of resilience as a product of environment and constitution that is an interactive process. Protective factors can modify a person's responses to an environmental hazard so that the outcome is not always detrimental and protective factors may only become detectable in the face of a stressor." [8, p. 20].

3 "Protective factors refer to conditions that improve people's resistance to risk factors and disorders. They have been defined as those factors that modify, ameliorate or alter a person's response to some environmental hazard that predisposes to a maladaptive outcome (Rutter [23]). Mostly, individual protective factors are identical to features of positive mental health, such as self-esteem, emotional resilience, positive thinking, problem-solving and social skills, stress management skills and feelings of mastery." [35, p. 20].

4 "Mental health is a fundamental element of the resilience, health assets, capabilities and positive adaptation that enable people both to cope with adversity and to reach their full potential and humanity." $[9$, p. III] .

5 "Mental health is defined as a state of well-being in which every individual realizes his or her own potential, can cope with the normal stresses of life, can work productively and fruitfully, and is able to make a contribution to her or his community." [10].
} 
Fig. 1 Annual numbers of papers published in European Child \& Adolescent Psychiatry that include the terms resilience and/or well-being. Search conducted in PubMed on 14-012016

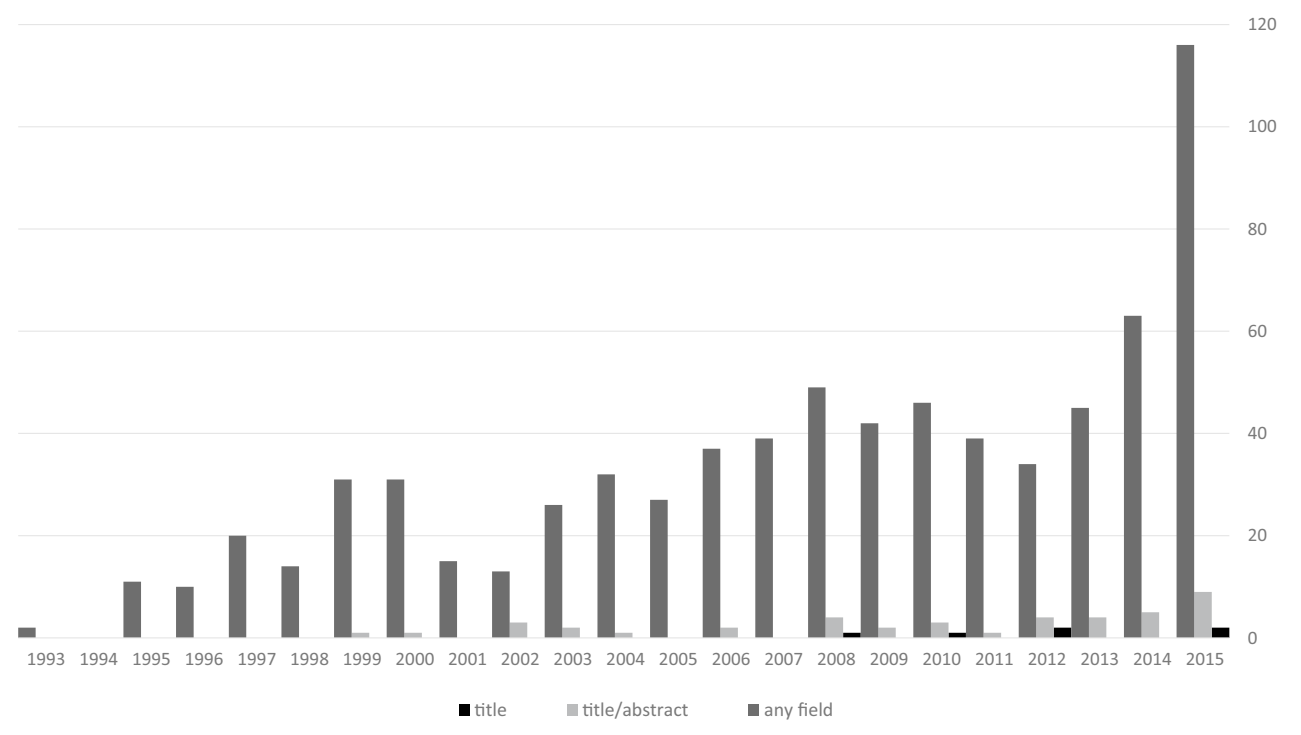

continuously criticized the lack of a universal definition of each construct and the indistinct use of both terms. Furthermore, the need for more research into these concepts in and across various contexts, such as children and adolescents, the elderly, patients, employees, or soldiers, has been repeatedly voiced [11-16]. As other concepts frequently co-occur with these terms, in particular risk, protective (or promotive) factors as well as mental health, such research should also take their similarities and differences into account when defining resilience and well-being.

\section{Resilience}

Resilience is an interactive phenomenon that is inferred from the observation that some individuals do well and better than others despite having experienced adversities [12]. A recent review of the definitions and conceptualizations of resilience in the health-related empirical literature published between January 2000 and April 2015 with a primary focus on resilience identified five overlapping, nonexclusive key themes [11]. Thereby resilience was alternatively described as follows:

- The process of overcoming (or thriving or succeeding) difficulties, adversity, or trauma to a point of becoming more successful or functioning even better than before (e.g., [12]).

- The process of adjustment and adaptation to new or difficult situations (e.g., [17]).

- The process of fully recovering (or bouncing back) from difficult periods or trauma (e.g., [17]).

- A form of mental immunity with good mental health as a proxy measure (e.g., [18]).
- A universal, yet difficult to quantify, personal strength grounded in positive experiences and support (e.g., [19]).

These five key themes can be viewed in light of two basic, nonexclusive perspectives on resilience that greatly influence its assessment [20]: the trait-oriented and the process-oriented perspective. The trait-oriented perspective regards resilience as an inborn ability or asset like a mental immunity, places responsibility on the person for overcoming adversity, and measures it similarly to other personal traits (such as personality traits) with self-report questionnaires or interviews irrespective of socioecological factors. The process-oriented perspective regards resilience as allostasis, i.e., as an interactive process between individuals and environment to achieve or maintain stability in the context of present or anticipated stressors through behavioural and/or physiological changes. Consequently, resilience is conceptualised not as a quality visible in every situation but as one defined and measured by the context, population, risk, promotive (or protective) factors, and outcome. In this latter, more commonly endorsed perspective, assets and resources are distinguished as two types of promotive factors that build resilience in a strict sense and that work on different levels [20-22]. Thereby, the term resources refers to presence of protective environmental factors and not merely to the absence of environmental risk factors, while the term assets refers to intrapersonal factors (Table. 1). Thus, in light of the process-oriented perspective, assets in terms of resilience only become apparent if challenged by extraordinary adversities and, consequently, should be distinguished from good coping abilities used in daily life to deal with normal levels of developmental stress. As a result, without a sufficient challenge, being 'problem-free' does not equal being 'fully able', i.e., resilient [22]. 


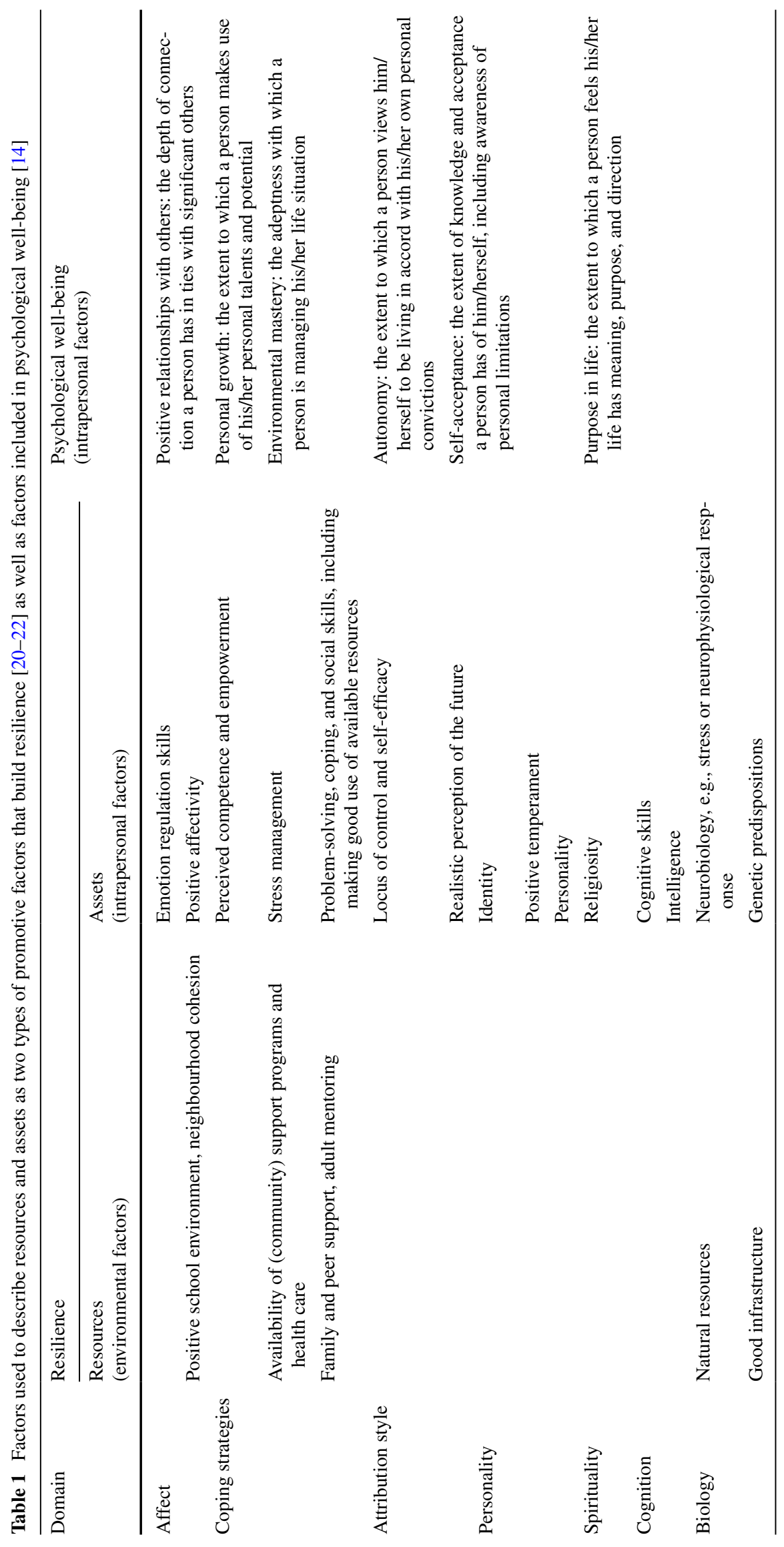




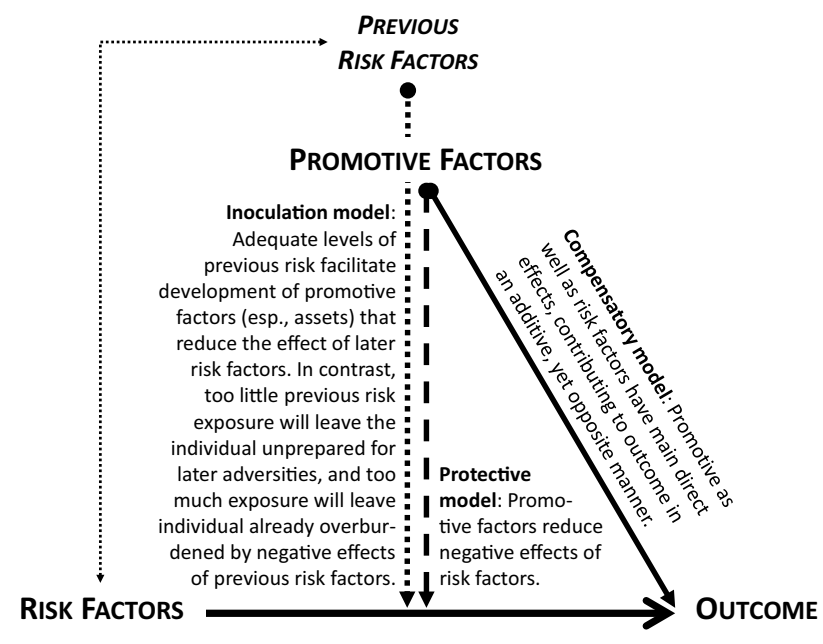

Fig. 2 The three basic models of the interplay of promotive and risk factors on outcome

Furthermore, three basic models of the mechanisms whereby promotive (protective) factors alter the relationship between risk exposure and outcome have been described [20]: (1) a compensatory model referring to an unidirectional direct and additive, yet opposite, effect of promotive and risk factors; (2) a prospective model referring to an interactive risk-modifying effect of promotive factors in terms of risk reduction; and (3) an inoculation model of a deferred reciprocal effect of risk and promotive factors (Fig. 2) captured by Nietzsche's quote, 'What does not kill me, makes me stronger' [23]. Whereas the compensatory model is compatible with both a trait- and a processoriented perspective, the prospective and the inoculation model both relate to the process-oriented perspective.

Given the apparent, possibly inherent difficulties in precisely framing or grasping the concept of resilience, its measurement is often indirect, using presence or absence of mental disorders or symptoms as a proxy despite the many other features related to resilience [11]. In a recent review, Ungar [22, p. 4] explicates this indirect method of measurement: 'Resilience is most commonly understood to be present if no disorder is diagnosed (exposure has had minimal impact), if disorder was previously present and the individual is recovering, or the individual exceeds expectations and is functioning better than before exposure to a potentially traumatizing event'. The absence of a 'gold standard', or even merely appropriate or adequate measurements [24] obstructs further clarification of this concept, thus creating a vicious circle that needs to be interrupted by more concept-related research. To address the complexity of the interaction between various promotive factors (assets and resources alike) and risk factors, development of a clinically useful evaluation tool of resilience and its sufficiency in light of a person's living situation will require large studies adopting a differential multidimensional approach. These should include neurobiological factors such as biological stress responses [25] and should take into account that it is unlikely that one 'diagnosis of resilience' will serve all [22]. Furthermore, future studies should also aim to specify possible core features of resilience across developmental stages, cultures and specific adverse living conditions such as war, migration, maltreatment, or living with a family member with severe mental illness, and the severity of adversities to be faced. This could be a starting point for an in-depth understanding of (sub)cultural variations in resilience and the pathways from resilience to positive outcome and well-being, as well as an important step towards positive psychiatry [26] and the development of preventive and resilience-enhancing treatments [12].

For the time being, a clinical impression of a child's or adolescent's resilience might be gained following the fivephase approach sketched by Ungar [22] and displayed in Table 2.

\section{Well-being}

In addition to mental health (i.e., the absence of mental disorders), well-being, also called wellness [15], is frequently used as an indicator of resilience, particularly when referring to resilience as a process of overcoming difficulties, adversity, or trauma so well that functioning is even better than before. Thereby, well-being extends beyond the absence of mental illness both psychologically and in terms of the involved genetic and environmental factors [27]. Challenging the view of well-being as a proxy of resilience, resilience assessed using a 10-item version of the Connor-Davidson Resilience Scale [28] was reported to be an independent, yet rather weak predictor of well-being, explaining only $12-19 \%$ of variance when controlling for personality factors (explaining 30-33\% of variance) and non-influential fluid intelligence. This finding might alternatively be regarded as support for the notion of well-being as a component of resilience [29].

Similar to the concept of resilience, no consensus on the definition or measurement of well-being has been reached so far $[13,15,16,30-33]$. For example, while the WHO has defined well-being as the 'presence of positive mental health ${ }^{6}[9$, p. 10], others have equated it to 'happiness' (e.g., [30]). More commonly, however, two related but distinct constructs of well-being are used today: subjective and psychological well-being $[16,30]$ :

\footnotetext{
6 "These outcomes are not just or necessarily a consequence of the absence of mental illness, but are associated with the presence of positive mental health, sometimes referred to as 'wellbeing'." [9, p. 10].
} 
Table 2 Five-phase decision tree for estimating resilience in children and adolescents according to Ungar [22]

Phase 1: Assessment of the exposure to adversity

Is there evidence of above normal, or atypical, exposure to adversity, or that the individual has experienced events in his or her life that threaten wellbeing, regardless of whether the individual shows mental health or behavioral problems?

\section{Yes}

Resilience is a relevant issue; continue assessment of Phase 2

\section{$\downarrow$}

No

Resilience is not a relevant issue.

$\rightarrow$

Shift focus of assessment from the individual's resilience to the individual's strengths.

Phase 2: Assessment of the impact of assets

Is the individual's abnormal exposure to risk excessively severe or chronic?

If yes, assessment of assets may not be sufficient to determine resilience. Resources should also be assessed.

If no, i.e., exposure to risk is normal or mild, then more emphasis should be placed on assessment of assets. Resources may still be important, but their influence is expected to be lower than at higher levels of exposure to risk. Sufficient assets may be enough to make individuals resilient in contexts of less severe and less chronic exposure to adversity.

\section{$\square \quad$ Yes}

Proceed to Phase 3 (resources) $\downarrow$

If need be, return to assessment of Phase 2 (assets)

thereafter

Phase 3: Assessment of the environment's resources

In all contexts where there are abnormal levels of adversity (high, medium and low), does the environment have the capacity to mitigate the impact of risk exposure?

To assess, review the availability and accessibility of resources, their strategic use, and whether the individual's assets when resources are used and assets judged favorably.

$\square \quad$ Yes

Environment has the capacity to sustain resilience.

Resilience is likely.
No

When resources are few, resilience is only likely if abnormal levels of adversity are low or medium, and if individual has sufficient assets.

If skipped earlier, return to Phase 2 (assets) before continuing Phase 4

Phase 4: Assessment of the self-perception of the sufficiency and adequacy of assets Are the individual's assets seen as adaptive by the individual? Depending on the social desirability of assets, and the individual's ability to influence the perception of others (help them to understand why a behavior is an appropriate response to adversity), the individual may be assessed as resilient or maladapted.

\section{Yes}

Assets are either experienced and/or perceived as adaptive. Resilience is likely. $\rightarrow$

Phase 5: Assessment of the contextual and cultural considerations regarding promotive factors Do the individual's assets meet their own, and/or others', expectations for how to behave under conditions of adversity?

If (a) maladaptive behaviors are reasonable given the environmental load and the availability and accessibility of resources, or (b) coping strategies reflect culturally relevant forms of adaptation that are reinforced by others, then a finding of (hidden, or culturally specific) resilience is appropriate.

Yes

Resilience is likely.

\section{No}

Assets may be temporarily functional. Resilience is not likely, however, in the longer term. 
Fig. 3 Core dimensions of psychological well-being according to Ryff [14] with brief descriptions of high scorers on each of these dimensions and, in Italics, the four dimensions of Seligman's PERMA model [34] resembling these core dimensions of $\mathrm{PWB}$

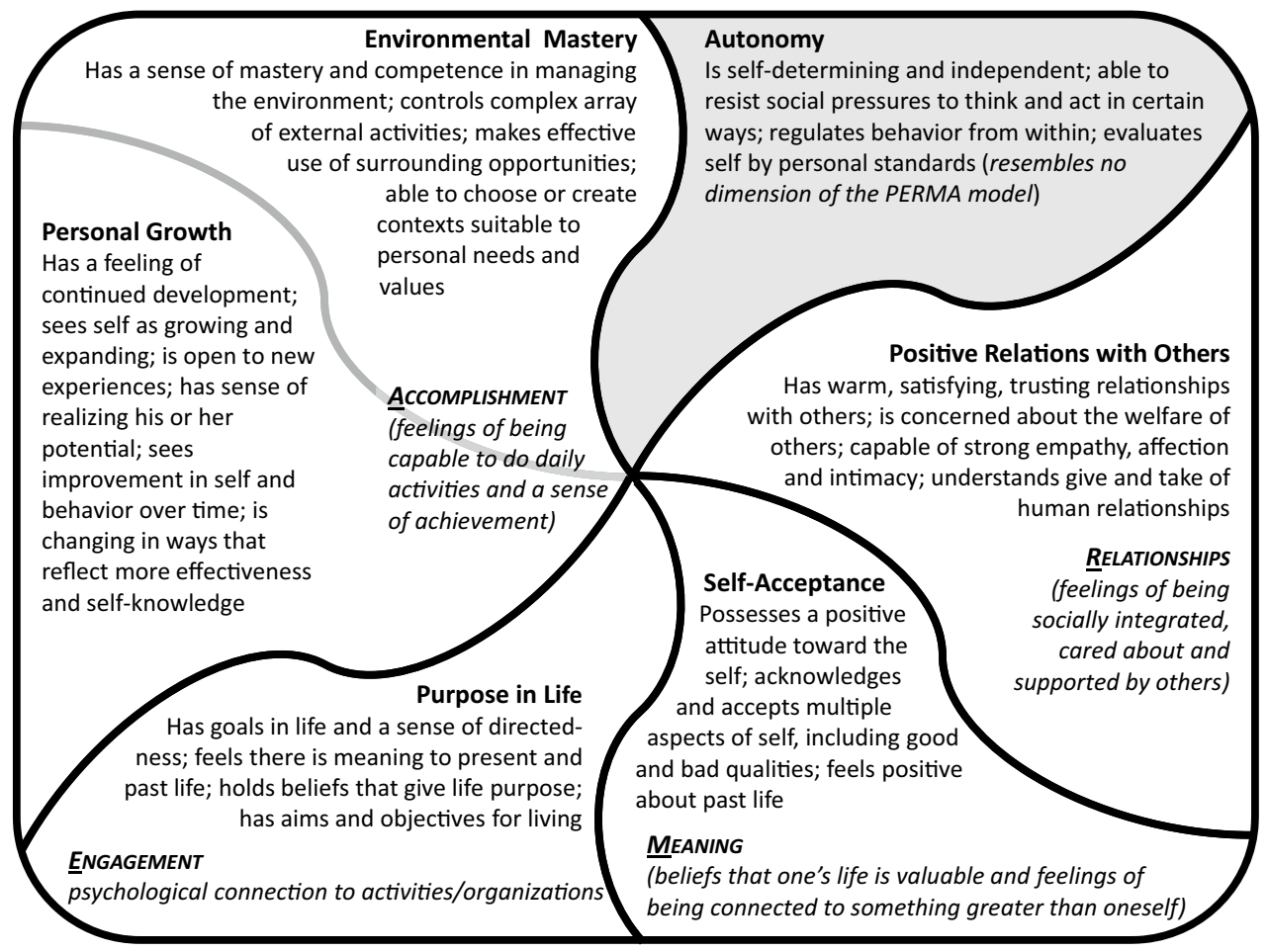

- Ryff's [14] psychological well-being (PWB), or eudaimonia from the Greek words 'eu' ('good') and 'daimōn' ('spirit'), often translated as 'happiness', 'welfare', or 'flourishing': PWB entails perception of engagement with existential challenges of life where well-being is seen as the full functioning of the person and a protective resource. It focuses on resources and strengths and is commonly represented by six factors that partly overlap with assets described in resilience research (Table. 1): purpose in life, autonomy, personal growth, environmental mastery, positive relationships with others, and self-acceptance (Fig. 3).

- Diener's [13] subjective well-being (SWB), or hedonia from the Greek word for 'delight' (hêdonismos from $h \bar{e} d o n \bar{e}$ 'pleasure'): SWB focuses on happiness and defines well-being in terms of pleasure attainment and pain avoidance. It consists of a cognitive component of judgement about one's life satisfaction (cognitive wellbeing) and an affective component characterized by the balance or preponderance of positive emotions relative to negative emotions (affective well-being).

A universal approach to well-being within the context of positive education was recently proposed by Seligman in terms of his five-dimensional PERMA model [34]. This includes Positive emotions, i.e., hedonic feelings of happiness that resemble the positive side of affective well-being in terms of SWB, as well as four dimensions related to PWB (Fig. 3): Engagement, Relationships, Meaning, and
Accomplishment. An initial study [34] supported the potential usefulness of this framework in measuring well-being of students in school settings important to adolescents' life satisfaction and positive mental health.

A systematic review of the definition of well-being, its measurement, and its common domains with an emphasis on paediatric literature [31] concluded that the concept was inconsistently defined with little agreement on its measurement. Thus, the authors suggested a definition of wellbeing as 'a complex, multi-faceted construct that has continued to elude researchers' attempts to define and measure' [31, p. 60]. Despite these shortfalls, five distinct domains of well-being were extracted from the literature [31]:

- A physical domain that includes physical health, rates of growth, and knowledge about eating healthily and staying safe.

- A psychological domain that comprises mental health, anxiety levels, and psychosocial aspects such as selfesteem, confidence, and emotions.

- A cognitive domain that involves aspects that are intellectual or school-related, including how children feel about school and their academic performance.

- A social domain that contains sociological perspectives such as family and peer relationships, communication skills, and the availability of emotional and practical support.

- An economic domain that includes family income and wealth, economic hardship, and availability of and 


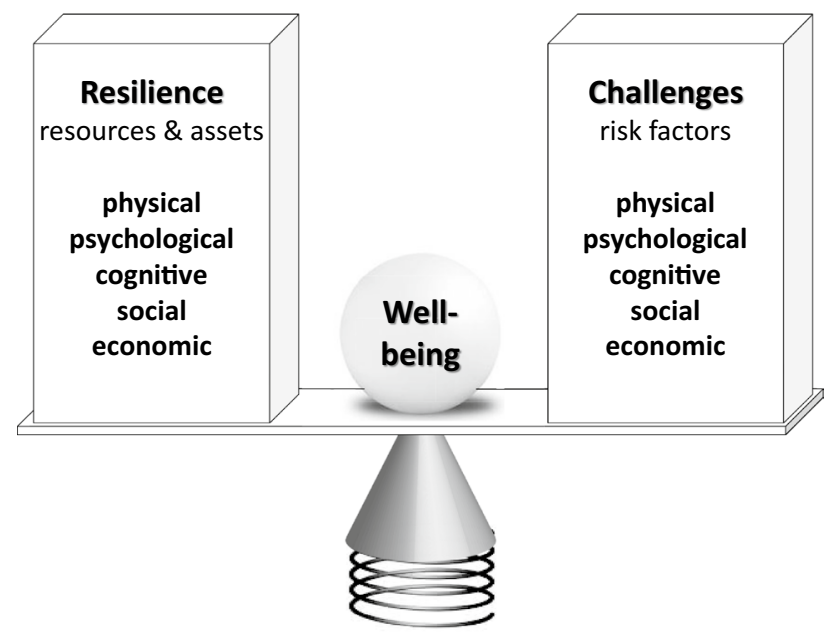

Fig. 4 Definition of subjective well-being according to [16] in light of the concept of resilience and accounting for long-term shifts in well-being (by the spiral)

access to economic support such as government benefit systems.

The elusiveness of a definition of well-being may be related to the fact that past research has been primarily driven by the identification of dimensions and by descriptions of well-being rather than by solidifying a definition [16]. Yet, despite the search for descriptions and dimensions, the multi-dimensionality of well-being continues to be often ignored, leading to narrow descriptions and unidimensional assessments, frequently quality of life or life satisfaction [16]. Thus, Dodge et al. [16] recently proposed a simple, universally applicable definition of (subjective) well-being as the 'balance point between an individual's resource pool and the challenges faced' [p. 230] that can easily accommodate various descriptions of domains and their operationalization and can nicely be linked to the concept of resilience (Fig. 4). Therein, SWB is regarded as an ever-changing rather than as a static concept, and the beam balance represents the person's drive to return to a set point of well-being, maintaining homeostasis. Thus, if resources (or resilience) are sufficient to meet a challenge (or risk), well-being will be relatively stable. However, if challenges overstrain resources, the beam balance-and, along with it, well-being-will dip. Moreover, a lack of challenges can lead to stagnation that will also affect balance and decrease well-being [16].

Since challenges might affect the various aspects of well-being differently, an adaptation of the definitional framework provided by Fig. 3 might be used for the differential consideration of affective and cognitive SWB. Furthermore, the base of the beam balance can change its height to account for long-term shifts in well-being, allowing for homeostasis at a new level. A recent meta-analysis of longitudinal studies of adaption to various life events and the effects on affective and cognitive SWB [32] challenged the notion of life events having no lasting effect on well-being, which is mainly supported by cross-sectional studies. Analysing the effect and adaptation curve of affective and cognitive SWB as well as eight life events separately, they found that life events affect both types of SWB differentially in terms of both the initial hedonic reaction and the rate of adaption, whereby most events initially and persistently affected cognitive SWB more negatively than affective SWB. Furthermore, affective SWB exhibited greater variance of effect sizes than cognitive SWB, indicating that the effects of life events on cognitive SWB are more consistent across samples. A possible explanation for the larger variance in changes of affective SWB might be the greater influence of other variables such as personality, coping strategies, mood regulation, or social support [32]or, in other words, of individual resilience.

Thus, despite its long tradition dating back to the ancient Greeks [13, 14], agreement on the definition, dimensions, and measurements of well-being has not yet been reached but warrants more empirical research.

\section{Conclusion}

Resilience and well-being have become popular terms and are central constructs in positive psychiatry and psychology [26]. However, both terms have so far defied universal definition and common understanding of their measurement. Furthermore, there is no general agreement on the relationship of the two constructs; while some use well-being as a proxy measure of resilience, others treat one concept as a component of the other, and yet another group views one concept as a prerequisite of the other. Part of the confusion around these two potentially valuable concepts is the overlap in their components, in particular with regard to resilience and psychological well-being, and the lack of research on these concepts both by themselves and in relation to each other or other related concepts [33]. Thus, more conceptual cross-sectional as well as longitudinal studies are clearly imperative (a) to uncover the composition of these constructs and to reach agreement on their definition and measurement, (b) to detect their potential neurobiological underpinnings, (c) to reveal how they relate to each other, and (d) to determine the potential role of developmental and cultural peculiarities. For the time being, however, the use of the terms resilience and wellbeing should always be accompanied by a brief explanation of their respective meanings and theoretical framework. 


\section{References}

1. Aebi M, Kuhn C, Metzke CW, Stringaris A, Goodman R, Steinhausen HC (2012) The use of the development and well-being assessment (DAWBA) in clinical practice: a randomized trial. Eur Child Adolesc Psychiatry 21(10):559-567. doi:10.1007/ s00787-012-0293-6

2. Barendregt CS, Van der Laan AM, Bongers IL, Van Nieuwenhuizen C (2015) Adolescents in secure residential care: the role of active and passive coping on general well-being and self-esteem. Eur Child Adolesc Psychiatry 24(7):845-854. doi:10.1007/ s00787-014-0629-5

3. Flouri E, Midouhas E, Joshi H, Tzavidis N (2015) Emotional and behavioural resilience to multiple risk exposure in early life: the role of parenting. Eur Child Adolesc Psychiatry 24(7):745-755. doi:10.1007/s00787-014-0619-7

4. Flouri E, Tzavidis N, Kallis C (2010) Adverse life events, area socioeconomic disadvantage, and psychopathology and resilience in young children: the importance of risk factors' accumulation and protective factors' specificity. Eur Child Adolesc Psychiatry 19(6):535-546. doi:10.1007/s00787-009-0068-x

5. Priebe G, Svedin CG (2012) Online or off-line victimisation and psychological well-being: a comparison of sexual-minority and heterosexual youth. Eur Child Adolesc Psychiatry 21(10):569582. doi:10.1007/s00787-012-0294-5

6. Ravens-Sieberer U (2008) The contribution of the BELLA study in filling the gap of knowledge on mental health and well-being in children and adolescents in Germany. Eur Child Adolesc Psychiatry 17(Suppl 1):5-9. doi:10.1007/s00787-008-1001-4

7. World Health Organization, WHO (2004) Promoting mental health. WHO, Geneva

8. World Health Organization, WHO (2003) Investing in mental heal th. WHO, Geneva

9. World Health Organization, WHO (2009) Mental health, resilience and inequalities. http://www.euro.who.int/_data/assets/ pdf_file/0012/100821/E92227.pdf. Accessed 2 February 2016

10. World Health Organization, WHO (2014) Mental health: a state of well-being. http://www.who.int/features/factfiles/mental_ health/en/. Accessed 1 January 2016

11. Aburn G, Gott M, Hoare K (2016) What is resilience? An integrative review of the empirical literature. J Adv Nurs. doi:10.1111/jan.12888

12. Rutter M (2013) Annual research review: resilience-clinical implications. J Child Psychol Psychiatry 54(4):474-487. doi:10.1111/j.1469-7610.2012.02615.x

13. Diener E (1984) Subjective well-being. Psychol Bull 95(3):542-575

14. Ryff CD (2014) Psychological well-being revisited: advances in the science and practice of eudaimonia. Psychother Psychosom 83(1):10-28. doi:10.1159/000353263

15. Kiefer RA (2008) An integrative review of the concept of well-being. Holist Nurs Pract 22(5):244-252. doi:10.1097/01. HNP.0000334915.16186.b2

16. Dodge R, Daly A, Huyton J, Sanders L (2012) The challenge of defining wellbeing. Int J Wellbeing 2(3):222-235. doi:10.5502/ ijw.v2i3.4

17. American Psychological Association (2016). The road to resilience. http://www.apa.org/helpcenter/road-resilience.aspx. Accessed 2 February 2016
18. Masten AS (2001) Ordinary magic: resilience processes in development. Am Psychol 56:227-238

19. Davydov DM, Stewart R, Ritchie K, Chaudieu I (2010) Resilience and mental health. Clin Psychol Rev 30:479-495. doi:10.1016/j.cpr.2010.03.003

20. Wang JL, Zhang DA, Zimmermann MA (2015) Resilience theory and its implications for Chinese adolescents. Psychol Rep 117:354-375. doi:10.2466/16.17.PRO.117c21z8

21. Fergus S, Zimmermann MA (2005) Adolescent resilience: a framework for understanding healthy development in the face of risk. J Adolesc Health 30:184-189

22. Ungar M (2015) Practitioner review: diagnosing childhood resilience-a systematic approach to the diagnosis of adaptation in adverse social and physical ecologies. J Child Psychol Psychiatry 56:4-17. doi:10.1111/jcpp. 12306

23. Nietzsche F (1997) Twilight of the idols [first published in 1889]. Hackett Publishing Company, Indianapolis

24. Ahern NR, Kiehl EM, Sole ML, Byers J (2006) A review of instruments measuring resilience. Issues Compr Pediatr Nurs 29(2):103-125. doi:10.1080/01460860600677643

25. Rutten BPF, Hammels C, Geschwind N, Menne-Lothmann C, Pishva E, Schruers K, van den Hove D, Kenis G, van Os J, Wichers M (2013) Resilience in mental health: linking psychological and neurobiological perspectives. Acta Psychiatr Scand 128:320. doi:10.1111/acps.12095

26. Jeste DV, Palmer BW, Rettew DC, Boardman S (2015) Positive psychiatry: its time has come. J Clin Psychiatry 76:675-683. doi:10.4088/JCP.14nr09599

27. Haworth CMA, Carter K, Eley T, Plomin R (2015) Understanding the genetic and environmental specificity and overlap between well-being and internalizing symptoms in adolescence. Dev Sci. doi:10.1111/desc. 12376

28. Campbell-Sills L, Stein MB (2007) Psychometric analysis and refinement of the Connor-Davidson Resilience Scale (CDRISC): validation of a 10-item measure of resilience. J Trauma Stress 20(6):1019-1028. doi:10.1002/jts.20271

29. Moreira PAS, Cloninger CR, Dinis L, Sá L, Oliveira JT, Dias A, Oliviera J (2015) Personality and well-being in adolescents. Front Psychol 5:1494. doi:10.3389/fpsyg.2014.01494

30. Linley PA, Maltby J, Wood AM, Osborne G, Hurling R (2009) Measuring happiness: the higher order factor structure of subjective and psychological well-being measures. Pers Indiv Diff 47:878-884. doi:10.1016/j.paid.2009.07.010

31. Pollard E, Lee P (2003) Child well-being: a systematic review of the literature. Soc Ind Res 61(1):59-78. doi:10.102 3/A:1021284215801

32. Luhmann M, Hofmann W, Eid M, Lucas RE (2012) Subjective well-being and adaptation ti life events: a meta-analysis. J Pers Soc Psychol 102(3):592-615. doi:10.1037/a0025948

33. Guérin E (2012) Disentangling vitality, well-being, and quality of life: a conceptual examination emphasizing their similarities and differences with special application in the physical activity domain. J Phys Act Health 9(6):896-908

34. Kern ML, Waters LE, Adler A, White MA (2015) A multidimensional approach to measuring well-being in students: application of the PERMA framework. J Pos Psychol 10(3):262-271. doi:10. 1080/17439760.2014.936962

35. World Health Organization, WHO (2004) Prevention of mental disorder. WHO, Geneva 